\title{
Fault Diagnosis of Wind Turbine Blades Based on Chaotic System and Extension Neural Network
}

\author{
Meng-Hui Wang, Cheng-Che Hsieh, and Shiue-Der Lu* \\ Department of Electrical Engineering, National Chin-Yi University of Technology, \\ No. 57, Sec. 2, Zhongshan Rd., Taiping Dist., Taichung 411030, Taiwan (R.O.C.)
}

(Received December 3, 2020; accepted February 22, 2021; online published April 14, 2021)

Keywords: chaos synchronization detection method, extension neural network, LabVIEW graphic control software, IEC 61850 communication protocol

We propose a chaos synchronization detection method combined with an extension neural network to diagnose the state of wind turbine blades. On the basis of a large-scale wind power generation system architecture, a $100 \mathrm{~W}$ small-scale wind power generation system simulation platform was first constructed and then a programmable logic controller (PLC) collected vibration sensor information. Through Ethernet and IEC 61850 communication protocols, the measured vibration signals were synchronously transmitted to a remote human-machine interface constructed by LabVIEW to facilitate remote real-time monitoring and analysis. We examined the identification of four different states of wind turbine blades: the normal state, blade rupture, blade screw fly-off, and abnormal blade inclination angle. On the basis of vibration signals in different states, a dynamic error scatter diagram was constructed by the chaos synchronization detection method, and chaos eye coordinates were extracted as eigenvalues for the identification of various state models. Finally, through the extension neural network, the four different states were identified. The measured results show that the proposed method can identify the states of wind turbine blades, and the identification accuracy rate of the proposed method was as high as $88.75 \%$. Therefore, the proposed method effectively detects abnormal vibration signals of wind turbines and identifies different types of blade faults in real time.

\section{Introduction}

The 2019 Global Wind Power Generation Report emphasized that in the next five years the global wind power industry will see rapid development. It is estimated that the annual growth rate of wind power generation will be $4 \%$, with capacity increasing by $355 \mathrm{GW}$ between 2020 and 2024, a rise of about $71 \mathrm{GW}$ per year. ${ }^{(1)}$ In addition, according to the 2020 World Energy Outlook issued by the International Energy Agency (IEA), renewable energy will meet $80 \%$ of the growth in the world's electricity demand in the next decade, and renewable energy such as wind power and solar power will supply nearly $40 \%$ of the world's electricity consumption in $2030 .^{(2)}$ In recent years, many enterprises around the world, such as the International Renewable

*Corresponding author: e-mail: sd1@ncut.edu.tw

https://doi.org/10.18494/SAM.2021.3208 
Energy Agency (IRENA), the Climate Group, and the Carbon Disclosure Project (CDP), have actively promoted the RE100 International Initiative, where participating enterprises must use renewable energy for all their electricity consumption by $2040 .{ }^{(3)}$ Throughout the report by IEA, it is shown that the world is now in an era of energy transition. In recent years, governments have proposed renewable energy power generation policies and have searched for and evaluated potential wind fields where wind power plants can be developed, so as to realize the vision of renewable energy power generation, such as wind power generation and solar power generation, and meet the above target of generating $40 \%$ of the world's power by 2030 .

Owing to the long-term exposure of wind turbines and their almost continuous operation, their parts undergo aging and wear, increasing the probability of faults. As a result, wind turbine system maintenance and fault diagnosis will be a major challenge for the industry in the future. Relevant wind power accident research reports have statistically analyzed the common wind turbine faults and their causes, including high nacelle temperatures, gearbox damage, blade damage, and bearing damage. ${ }^{(4)}$ According to statistics on wind turbine failure shutdown, among the large components, blades account for the largest proportion of faults (35\%), followed by dynamos (32\%), gearboxes (21\%), and other components (8\%). ${ }^{(5)}$ Among the wind turbine operation and maintenance expenses, blade operation and maintenance expenses make up the largest proportion (30\%), followed by dynamos (28\%) and gearboxes $(19 \%){ }^{(5)}$ Therefore, to reduce the impact of current wind turbine failures on the system, blade faults should be focused on as the top priority. If a fault diagnosis technique for blades can be developed, then in the case of a minor abnormality, such a technique can be used for detection in real time, which would effectively reduce the fault time of wind turbines, increase the availability rate, and reduce operation and maintenance costs.

In previous research on blade condition detection, Yang et al. proposed a method based on frequency index calculation for blade vibration dynamics and frequency analysis, which was applied to 29-inch glass fiber low-wind-speed dynamo blades. If the blades are ruptured, then the rupture position on the damaged blades can be clearly identified through this method. ${ }^{(6)}$ Wang and Zhang put forward a data-driven framework and Haar-like method to screen test images and locate damaged blade areas through window scaling. The blade images were collected by a remote-controlled unmanned aerial vehicle and the surface cracks of blades were detected. ${ }^{(7)}$ Zhang et al. proposed the analysis of aeroacoustic characteristics and the modeling of acoustic signal calculation noise in accordance with the IEEE P2400 standard to measure the aerodynamic noise changes of a blade airfoil surface through a voiceprint sensor and to diagnose blade rupture. ${ }^{(8)}$ Malik and Mishra proposed a wind turbine state monitoring method based on an artificial neural network and an empirical mode decomposition method to evaluate whether aerodynamic parts such as the blades, nacelle, tail, and rotor are in equilibrium and a stable state. ${ }^{(9)}$

In the above literature, most scholars emphasized the state of blade surface cracks and rarely studied mechanical faults such as a blade screw flying off or an abnormal blade inclination angle. Therefore, in the present study, we use a programmable logic controller (PLC) and an analog-signal-capturing module to capture the electrical signals of the vibration sensor of a wind turbine simulation platform. In addition, a remote monitoring system is constructed with 
LabVIEW graphic control software that integrates the IEC 61850 communication protocol, Ethernet, a chaos synchronization detection method, and an extension neural network (ENN) to identify the most common blade states: the normal state, blade screw fly-off, blade rupture, and an abnormal blade inclination angle. The measured results show that the identification accuracy rate of the proposed method is as high as $88.75 \%$. Therefore, the online fault diagnosis system for wind blades developed in this paper may be applied to actual wind fields in the future to improve the operation and maintenance capability of overall wind turbine systems.

\section{Wind Turbine Blade Fault Vibration Signal Measurement}

In this study, the fault diagnosis of wind power generation blades has three main parts: a $100 \mathrm{~W}$ wind power generation system test platform, vibration signal measurement, and a human-machine interface for remote monitoring and fault diagnosis, as shown in Fig. 1. First, the vibration signals of a wind turbine are measured by sensors, and a PLC and analog input converter are used as a system to collect sensor signals. The IEC 61850 communication protocol is employed as a data transmission medium between the PLC and LabVIEW, thus realizing a human-machine interface. Finally, the algorithm proposed in this paper is imported into the human-machine interface to develop a remote real-time information and fault detection system.

\subsection{Test platform for small-scale wind power generation system}

In this study, a large-scale wind turbine structure is modeled with a small-scale wind power generation system test platform, as shown in Fig. 2. For the main configuration, the blades of a wind turbine and a single-phase drive motor are used as simulated wind energy to drive the blades, a speed increaser is adopted to simulate the gearbox inside the wind turbine nacelle, and then the energy is transmitted to the generator and supplied to the load. The vibration signals of the blade bearings are captured through vibration sensors. Table 1 lists the detailed specifications of each component of the test platform for the wind power generation system.

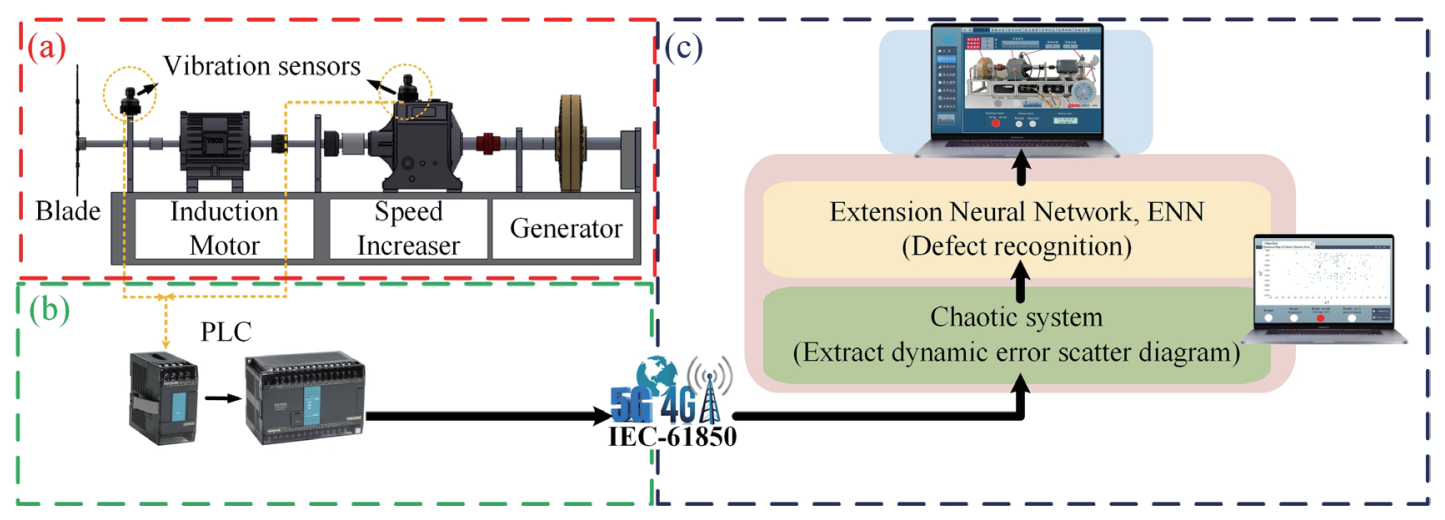

Fig. 1. (Color online) Overall architecture of wind power generation fault diagnosis system: (a) Wind power system test platform, (b) electrical signal measurement and PLC, and (c) human-machine interface for fault diagnosis. 


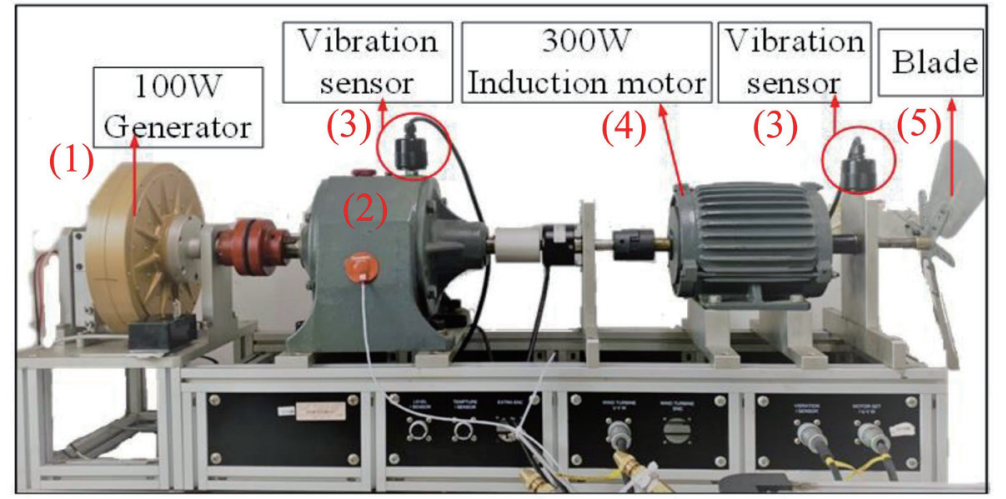

Fig. 2. (Color online) $100 \mathrm{~W}$ test platform for wind power generation system.

Table 1

Detailed specifications of each component of test platform for wind power generation system.

\begin{tabular}{lcc}
\hline Number & Component name & Specification \\
\hline$(1)$ & Generator & $100 \mathrm{~W}$ \\
\hline$(2)$ & Gearbox & Gear ratio $1: 15$ \\
\hline$(3)$ & Vibration sensor & $\begin{array}{c}\text { Full scale range: } 50 \mathrm{~mm} / \mathrm{s} \mathrm{RMS} \\
\text { Output current: } 4 \text { to } 20 \mathrm{~mA}\end{array}$ \\
\hline$(4)$ & Three-phase induction motor & $220 \mathrm{~V} / 1 \mathrm{HP}$ \\
\hline$(5)$ & Wind turbine blade & $30 \mathrm{~cm}$ \\
\hline
\end{tabular}

To explore the abnormal vibration of wind turbine blades when faults occur, a normal blade model (State I) and three common blade fault models (States II-IV) are established through the constructed wind turbine simulation test platform. We then discuss the vibration phenomena caused by different states of the wind turbine blades.

\section{A. Normal wind turbine blade (State I)}

On the basis of the blade structure of a large wind turbine, a small-scale blade is constructed on the test platform in this study. Figure 3 illustrates the model of the blade under a normal state.

\section{B. Blade rupture (State II)}

Most wind turbines operate in a harsh environment, where they are vulnerable to strong wind, bird impact, or lightning strikes caused by a failure in the lightning protection system, resulting in damage or rupture of the blades. Therefore, we construct a blade rupture model to simulate blade rupture caused by external factors, as shown in Fig. 4.

\section{Blade screw fly-off (State III)}

As wind turbines operate outdoors for a long time, their screws may gradually loosen due to the impact of external factors, resulting in fly-off or even breaking of the screws. If such a 


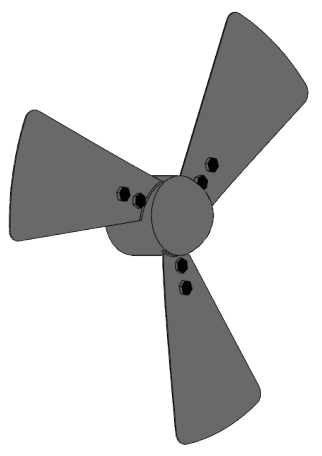

Fig. 3. (Color online) Model of blade under normal state.

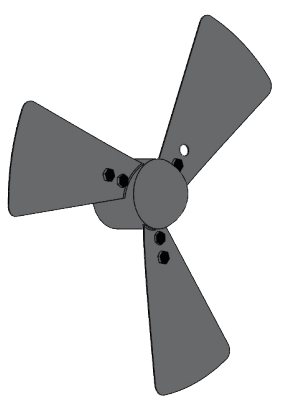

Fig. 5. (Color online) Model of blade screw flyoff.

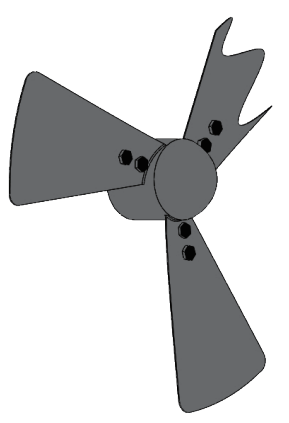

Fig. 4. (Color online) Model of wind turbine blade rupture.

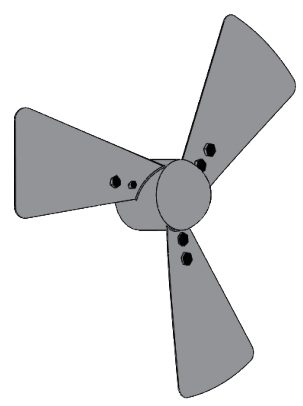

Fig. 6. (Color online) Model of abnormal blade inclination angle.

problem is not identified in a timely manner, then it will cause the blade to fly off. Therefore, we construct a model of blade screw fly-off to simulate screw fly-off caused by external factors, as shown in Fig. 5.

\section{Abnormal blade inclination angle (State IV)}

Considering the blade pitch control of large wind turbines, long-term operation often causes mechanical damage to the motor or internal gear differences, resulting in an unbalanced body caused by different wind angles of blades. Therefore, we establish a model of abnormal blade inclination angle to simulate a blade inclination angle fault of wind turbines, as shown in Fig. 6.

\subsection{Measurement of vibration signals}

We made four groups of blades with different states and carried out experiments on a $100 \mathrm{~W}$ wind power test platform, using a vibration sensor (SHOWA 2502-03) for measurement. The range of vibration displacement was $0-200 \mu \mathrm{m}$ and the output signal was a 4-20 mA analog signal. The data were collected through the PLC [Fig. 1(b)], and the vibration signal was captured at a sampling frequency of $2 \mathrm{~Hz}$. The vibration signal detected after the actual operation in each 
state is shown in Fig. 7 (State I: normal wind turbine blade, State II: blade rupture, State III: blade screw fly-off, State IV: abnormal blade angle location). The sampling time of the captured vibration signal was $300 \mathrm{~s}$ and the data length was $600 \mathrm{bits}$. After measuring the vibration of the faulty blade model, data were transmitted through the IEC 61850 communication protocol and Ethernet network, and the human-machine interface to detect and analyze the vibration signals was designed using LabVIEW software.

\subsection{Human-machine interface for remote monitoring and fault diagnosis [Fig. 1(c)]}

To develop a wind power generation fault diagnosis system, we integrate the proposed method into a graphic control human-machine interface developed using LabVIEW software [Fig. 1(c)]. The system mainly transmits the blade vibration signal data captured by the PLC to the remote monitoring interface through the IEC 61850 communication protocol and wireless network unit, as shown in Fig. 8. For fault diagnosis, the features of vibration signals are

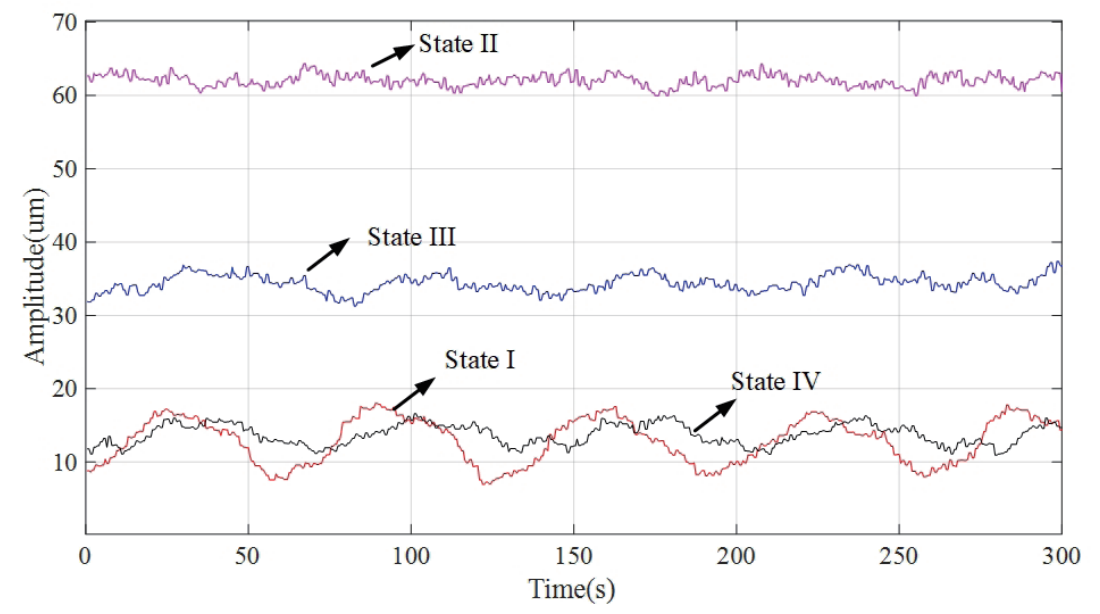

Fig. 7. (Color online) Measured vibration signals for different states of wind turbine blades.

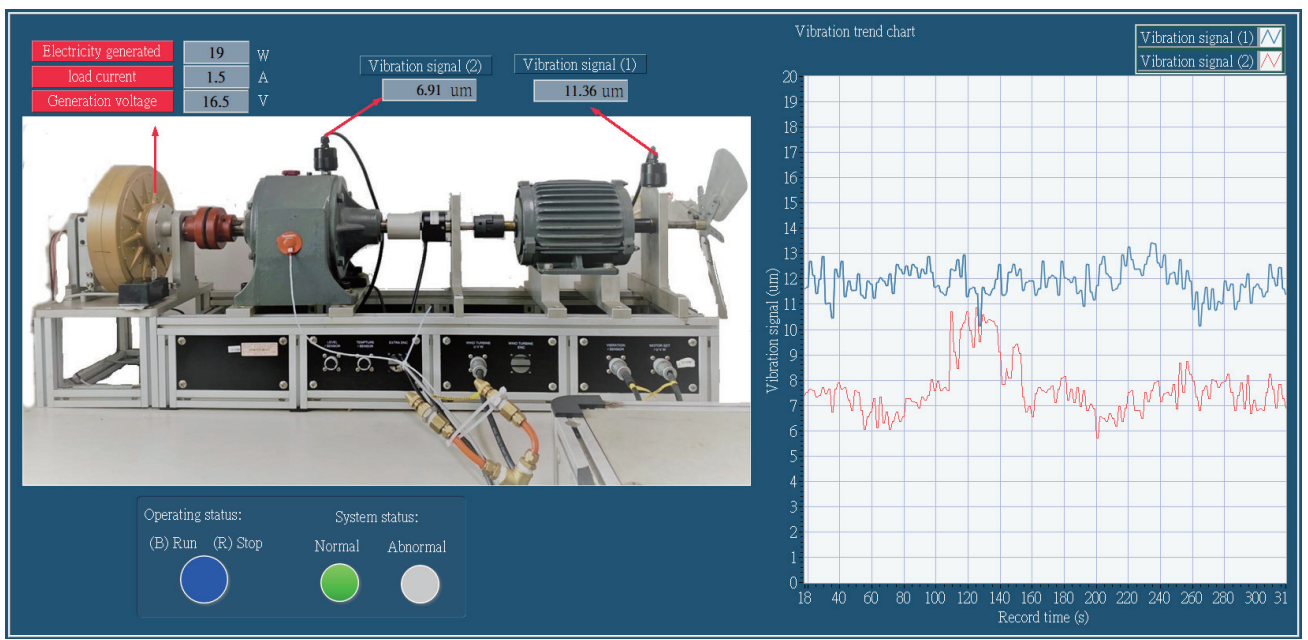

Fig. 8. (Color online) Human-machine interface for blade surveillance system. 
extracted by using the interface to plot a dynamic error scatter diagram of a chaotic system, denoted by (A) in Fig. 9. Finally, a trained ENN model is used for fault identification, and the diagnosis results are displayed on the human-machine interface [(B) in Fig. 9].

\section{Methodology}

We propose a chaos synchronization detection method combined with an ENN to identify blade faults. The flow chart of system identification is shown in Fig. 10. First, through Ethernet and IEC 61850 communication protocols, the vibration signals captured by the PLC are transmitted to the remote monitoring terminal, and the remote monitoring interface constructed by LabVIEW presents the relevant signals. Second, the measurement signals are converted into a synchronization error scatter diagram through the Lorenz chaos synchronization detection method, and the coordinate values of the left and right barycenters of the chaos eyes of the scatter diagram are extracted as characteristic signals of the state. Finally, the ENN is employed to learn and identify the signal features of the four states.

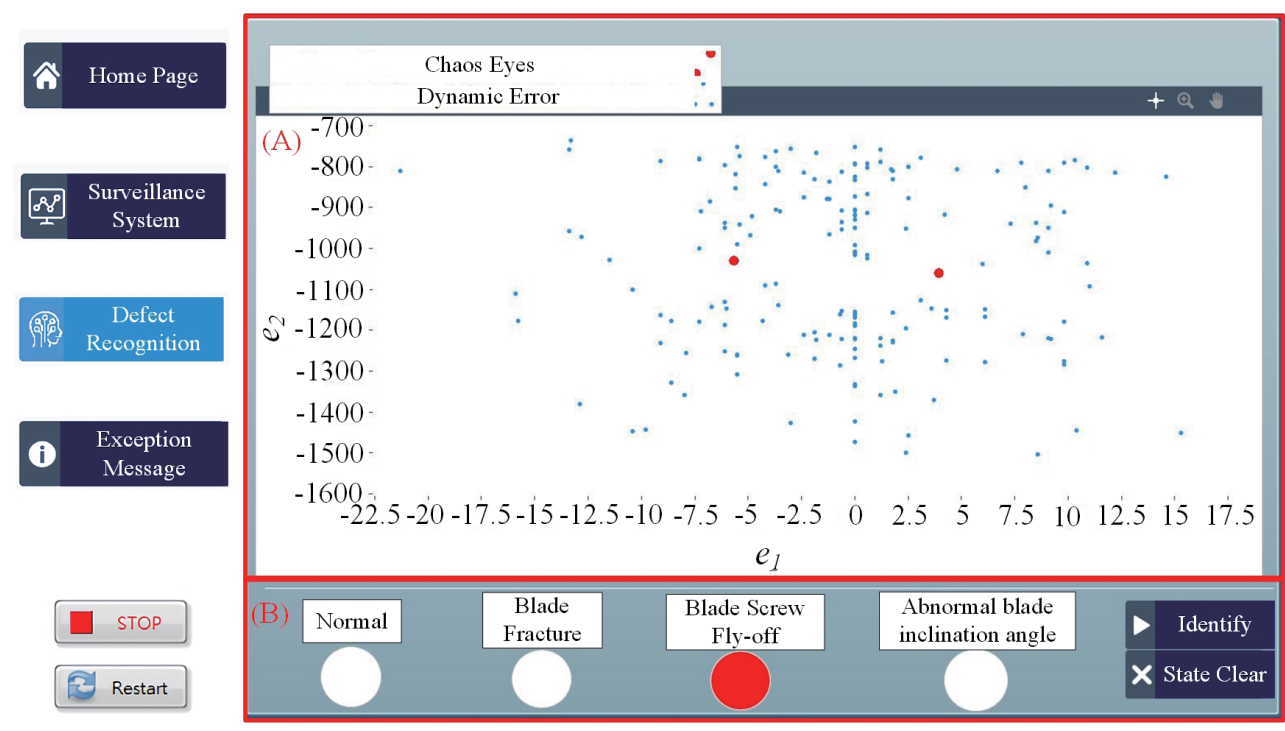

Fig. 9. (Color online) Human-machine interface for blade surveillance system.

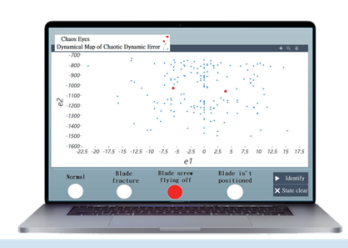

IoT remote monitoring system (Receive signal)

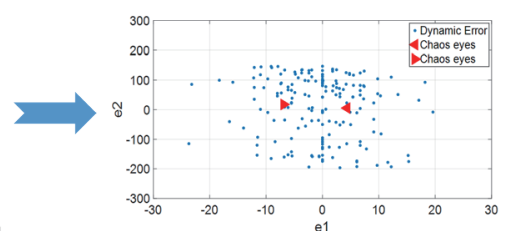

Chaotic system (Extract dynamic error scatter diagram)

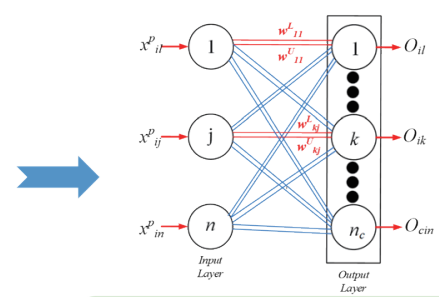

Extension neural network (Defect recognition)

Fig. 10. (Color online) Flow chart of data feature extraction and identification system. 


\subsection{Chaos synchronization detection method}

For signal feature extraction, we use the chaos synchronization detection method to convert the captured vibration signals into synchronization error scatter diagrams and adopt the barycentric coordinates (chaos eyes) of the scatter diagrams for the feature extraction of fault signals. This method has often been applied to fault signal feature extraction. ${ }^{(10,11)}$ Chaos theory was proposed by Edward Norton Lorenz, an American meteorologist, and the chaos phenomenon is a characteristic of nonlinear systems. We utilize the chaotic system of Lorenz for system feature extraction. ${ }^{(12)}$ The signal generated through the chaotic system generates an ordered but non-periodic motion trajectory due to a strange attractor, and this motion trajectory varies greatly upon minor changes. In a chaotic synchronization signal system, the chaotic system has a master system (MS) and a slave system (SS). When the two systems receive different signals, the motion trajectory of the two systems will produce different dynamic errors, and the SS will track the MS. This is called chaotic synchronization action. ${ }^{(13)}$ We mainly use the dynamic errors generated in the tracking process of the master-slave system to analyze the differences caused by different vibration signals.

The dynamic error generated by a vibration signal measured in this study can be plotted as a dynamic error scatter diagram, as shown in Fig. 11, by the chaos synchronization detection method. The dynamic error scatter diagram presents different distribution conditions and different barycenter positions for different blade states.

Equations (1) and (2) show the representation of the MS and SS of a chaotic system, respectively.

$$
\text { Master System }=\left\{\begin{array}{c}
\dot{x}_{1}=f_{1}\left(x_{1}, x_{2}, x_{3}, \ldots, x_{n}\right) \\
\dot{x}_{2}=f_{2}\left(x_{1}, x_{2}, x_{3}, \ldots, x_{n}\right) \\
\vdots \\
\dot{x}_{n}=f_{n}\left(x_{1}, x_{2}, x_{3}, \ldots, x_{n}\right)
\end{array}\right.
$$

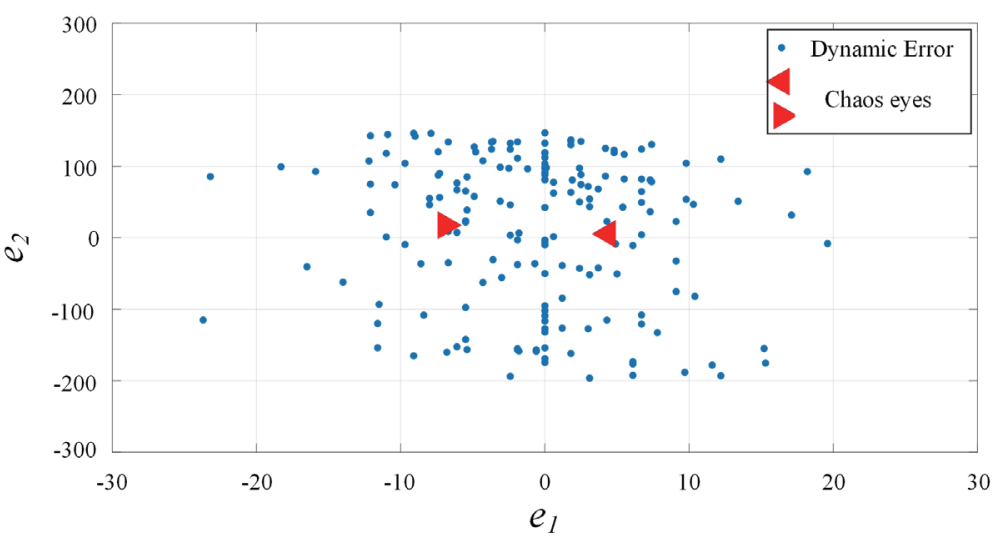

Fig. 11. (Color online) Chaotic dynamic error scatter diagram. 


$$
\text { Slave System }=\left\{\begin{array}{c}
\dot{y}_{1}=f_{1}\left(y_{1}, y_{2}, y_{3}, \ldots, y_{n}\right) \\
\dot{y}_{2}=f_{2}\left(y_{1}, y_{2}, y_{3}, \ldots, y_{n}\right) \\
\vdots \\
\dot{y}_{n}=f_{n}\left(y_{1}, y_{2}, y_{3}, \ldots, y_{n}\right)
\end{array}\right.
$$

Here, $x$ and $y$ are state vectors and $f(x)$ and $f(y)$ are nonlinear functions. Subtracting Eqs. (1) and (2) will give the master-slave dynamic errors, such as those in Eq. (3). The calculated dynamic error equation is shown in Eq. (4).

$$
\begin{gathered}
\left\{\begin{array}{c}
e_{1}=x_{1}-y_{1} \\
e_{2}=x_{2}-y_{2} \\
\vdots \\
e_{n}=x_{n}-y_{n}
\end{array}\right. \\
\left\{\begin{array}{c}
e_{1}=f_{1}\left(x_{1}, x_{2}, x_{3}, \ldots, x_{n}\right)-f_{1}\left(y_{1}, y_{2}, y_{3}, \ldots, y_{n}\right) \\
e_{2}=f_{2}\left(x_{1}, x_{2}, x_{3}, \ldots, x_{n}\right)-f_{2}\left(y_{1}, y_{2}, y_{3}, \ldots, y_{n}\right) \\
\quad \vdots \\
e_{n}=f_{n}\left(x_{1}, x_{2}, x_{3}, \ldots, x_{n}\right)-f_{n}\left(y_{1}, y_{2}, y_{3}, \ldots, y_{n}\right)
\end{array}\right.
\end{gathered}
$$

We employ the Lorenz chaotic system to extract the characteristics of the vibration signals of the four blade states. Equations (5) and (6) respectively present the MS and SS of the Lorenz chaotic system.

$$
\begin{gathered}
\text { Lorenz Master System }=\left\{\begin{array}{l}
\dot{x}_{1}=\alpha\left(x_{2}-x_{1}\right) \\
\dot{x}_{2}=\beta x_{1}-x_{1} x_{3}-x_{2} \\
\dot{x}_{3}=x_{1} x_{2}-\gamma x_{3}
\end{array}\right. \\
\text { Lorenz Slave System }=\left\{\begin{array}{l}
\dot{y}_{1}=\alpha\left(y_{2}-y_{1}\right) \\
\dot{y}_{2}=\beta y_{1}-y_{1} y_{3}-y_{2} \\
\dot{y}_{3}=y_{1} y_{2}-\gamma y_{3}
\end{array}\right.
\end{gathered}
$$

By subtracting Eqs. (5) and (6), the dynamic error equation of the Lorentz master-slave chaotic system can be obtained, expressed in matrix form in Eq. (7). Here, $\alpha, \beta$, and $\gamma$ are system parameters. Following Edward Lorenz, we set them as $\alpha=10, \beta=28$, and $\gamma=8 / 3$ to ensure that the system has the characteristics of strange attractors. ${ }^{(14)}$

$$
\left[\begin{array}{l}
\dot{e}_{1} \\
\dot{e}_{2} \\
\dot{e}_{3}
\end{array}\right]=\left[\begin{array}{ccc}
-\alpha & \alpha & 0 \\
\beta & -1 & 0 \\
0 & 0 & -\gamma
\end{array}\right]\left[\begin{array}{l}
e_{1} \\
e_{2} \\
e_{3}
\end{array}\right]+\left[\begin{array}{c}
0 \\
-e_{1} e_{3} \\
e_{1} e_{2}
\end{array}\right]
$$




\subsection{ENN}

Data identification is used in various research fields, mostly in fault diagnosis, disease diagnosis, data identification, and other applications. ${ }^{(15-18)}$ When the system characteristic parameters are too complex, AI methods are used to assist fault diagnosis. We adopt an ENN for fault diagnosis.

\section{A. Architecture of ENN}

There have been many studies on the successful application of ENNs in fault diagnosis. ${ }^{(19-21)}$ Figure 12 is the architecture diagram of an ENN, including the input layer and output layer. First, the input data are classified and built into a matter-element model and then imported into the ENN. The number of input layers is determined by the number of features of the matterelement model, and each input node and output node have two weight connections. One weight represents the minimum value of the input characteristics of this node, and the other weight represents the maximum value of the input characteristics of this node. For example, the $j$ th input node and $k$ th output node connect $W_{k j}^{L}$ and $W_{k j}^{U}$. The output layer is determined by the number of data categories and stores the calculated extension distance. The data category is determined by the minimum extension distance of the output layer belonging to each category.

\section{B. Learning method of ENN}

The learning methods of ENNs are divided into unsupervised learning and supervised learning. We propose to adjust the weight by the latter method, and adjust and correct the weight by continuous learning and training to achieve the same output value as the target or to reduce the error of the output value. There are several variables that need to be defined before learning.

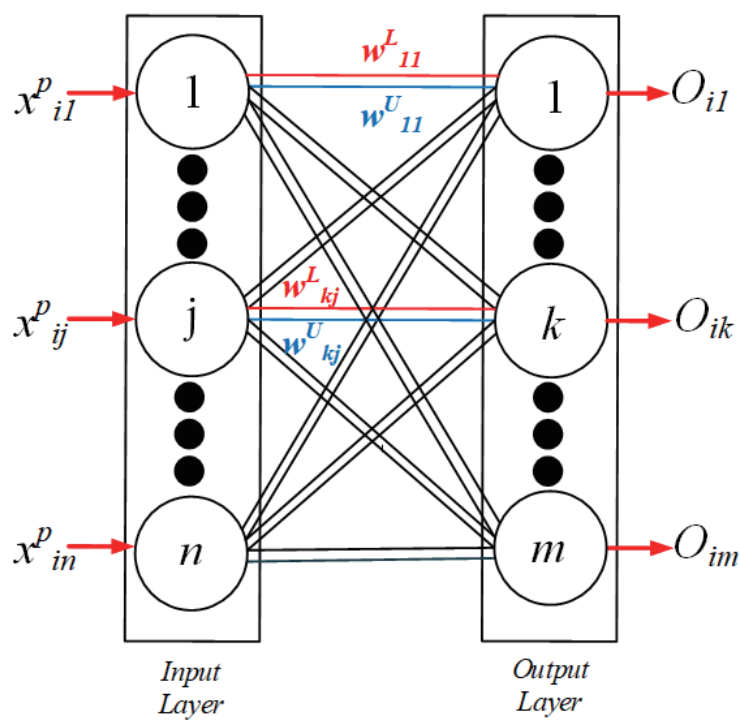

Fig. 12. (Color online) Architecture diagram of ENN. 
The learning sample is $x=\left\{x_{1}, x_{2}, \ldots, x_{N_{n}}\right\}$ and $N_{p}$ is the total number of training samples. The $i$ th sample is $x_{i}^{p}=\left\{x_{i 1}^{p}, x_{i 2}^{p}, \ldots, x_{i n}^{p}\right\}$, the total number of characteristic samples is $n$, and the category of the $i$ th sample is $p$. To evaluate the correctness of the ENN prediction, the total test error is set to $N_{m}$ and the total error ratio is set to $E_{T}$, where

$$
E_{T}=\frac{N_{m}}{N_{p}}
$$

The calculus steps of supervised learning for the ENN are as follows.

Step 1: Set the weight value between the connection input node and the output node according to the characteristic model as shown in Eqs. (9) and (10).

$$
\begin{gathered}
W_{k j}^{L}=\min _{i \in P_{N}}\left\{X_{i j}^{k}\right\} \\
W_{k j}^{U}=\max _{i \in P_{N}}\left\{X_{i j}^{k}\right\}
\end{gathered}
$$

Step 2: Calculate the weight center value of each feature and express it as $Z_{k}$.

$$
\begin{gathered}
Z_{k}=\left\{Z_{k 1}, Z_{k 2}, Z_{k 3}, \cdots, Z_{k n}\right\} \\
Z_{k j}=\frac{\left(W_{k j}^{L}+W_{k j}^{U}\right)}{2}
\end{gathered}
$$

Here, $k=1,2, \ldots, n ; j=1,2, \ldots, n$.

Step 3: Read the $i$ th sample data and feature number $p$, as shown in Eq. (13):

$$
X_{i}^{p}=\left\{X_{i 1}^{p}, X_{i 2}^{p}, X_{i 3}^{p}, \cdots, X_{i n}^{k}\right\}, p \in m
$$

Here, $X_{i n}^{p}$ represents the input learning data.

Step 4: $X_{i n}^{p}$ is used to calculate the extension distance and the distance between the sample and the $k$ th cluster, expressed as

$$
E D_{i k}=\left[\sum_{j=1}^{n} \frac{\left|X_{i j}^{k}-Z_{k j}\right|-\frac{\left(W_{k j}^{U}-W_{k j}^{L}\right)}{2}}{\left|\frac{\left(W_{k j}^{U}-W_{k j}^{L}\right)}{2}\right|}+1 .\right.
$$

Here, $k=1,2, \ldots, m$. 
Step 5: Find the data category of test results $k^{*}, E D_{i k^{*}}=\min _{k \in m}\left\{E D_{i k}\right\}$. If $k^{*}=p$ then jump to Step 7 . If the data categories are not equal, then $k^{*}=k$ and repeat Step 6. Figure 13 is the extension distance graph.

Step 6: Adjust the upper and lower limit weight values and weight center values for the $k$ th feature and $k^{*}$ th cluster.

(a) Update the upper and lower limit weight values of the $k$ th feature $k^{*}$ th cluster.

$$
\begin{gathered}
W_{k j_{-} \text {new }}^{L}=W_{k j_{-} \text {old }}^{L}+\eta\left(X_{i j}^{k}-Z_{k j_{-} \text {old }}\right) \\
W_{k j_{-} \text {new }}^{U}=W_{k j_{-} \text {old }}^{U}+\eta\left(X_{i j}^{k}-Z_{k_{j_{-}} \text {old }}\right) \\
W_{k^{*} j_{-} \text {new }}^{L}=W_{k^{*} j_{-} \text {old }}^{L}-\eta\left(X_{i j}^{k}-Z_{k^{*} j_{-} \text {old }}\right) \\
W_{k^{*} j_{-} \text {new }}^{U}=W_{k^{*} j_{-} \text {old }}^{U}-\eta\left(X_{i j}^{k}-Z_{k^{*} j_{-} \text {old }}\right)
\end{gathered}
$$

Here, $\eta$ is the learning rate.

(b) Update the weight center values of the $k$ th feature and $k^{*}$ th cluster.

$$
\begin{aligned}
Z_{k j_{-} \text {new }} & =\frac{\left(W_{k j_{-} n e w}^{U}+W_{k j_{-} n e w}^{L}\right)}{2} \\
Z_{k^{*} j_{-} \text {new }} & =\frac{\left(W_{k^{*} j_{-} \text {new }}^{U}+W_{k^{*} j_{-} \text {new }}^{L}\right)}{2}
\end{aligned}
$$

Step 7: Repeat Steps 3-6 until all learning data are read and the learning classification is completed.

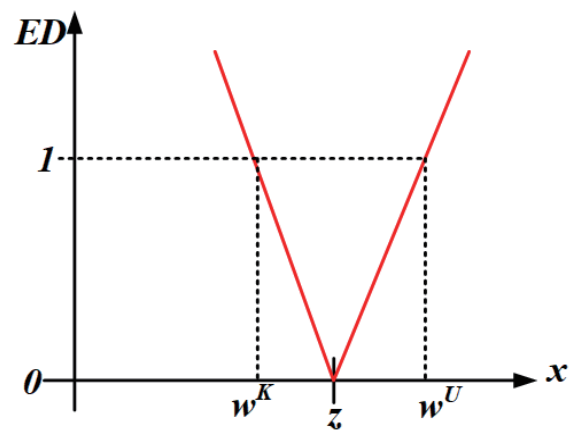

Fig. 13. (Color online) Extension distance graph. 
Step 8: Stop when the classification program of all data has reached the converged state or the total error rate has reached the target value; otherwise, return to Step 3.

\section{Identification calculus process of ENN}

When the ENN completes the learning program, it can be identified. Its calculation flow is shown in Fig. 14. When all samples are identified, the operation will stop; otherwise, the program returns to Step 3 to read the next test sample.

\section{Experimental Results}

There are four states of wind turbine blades in this study, 200 bits of vibration signal data of the blades are captured, and 50 measurements are made for each model. After calculation by the Lorenz chaotic system, each model has 50 groups of chaotic barycentric coordinate values, 30 of which are randomly selected as training samples and the remaining 20 are used as test samples. Therefore, there are 120 groups of training samples and 80 groups of test samples for the four states, which are used as data for the subsequent ENN calculus.

\subsection{Chaotic dynamic error scatter diagram}

The measured vibration signals of the models of wind turbine blades are input into the Lorenz chaotic system for dynamic error calculation, and three types of dynamic error scatter diagram are compiled, $e_{1} e_{2}, e_{1} e_{3}$, and $e_{2} e_{3}$, as shown in Fig. 15. The three types of dynamic error scatter diagram show different distributions, and so the barycenter positions (red labels in Fig. $15)$ are different. Hence, in this study, the barycenter coordinates $\left(e_{1} e_{2}, e_{1} e_{3}, e_{2} e_{3}\right)$ of the three types of dynamic error scatter diagram are trained and recognized by ENNs in order to compare their performance and to extract the optimal dynamic error. The analysis results are shown in Table 2. The chaotic barycenter coordinates obtained from the dynamic error scatter diagrams $e_{1} e_{2}, e_{1} e_{3}$, and $e_{2} e_{3}$ are also used to plot the barycenter distribution map of the models for

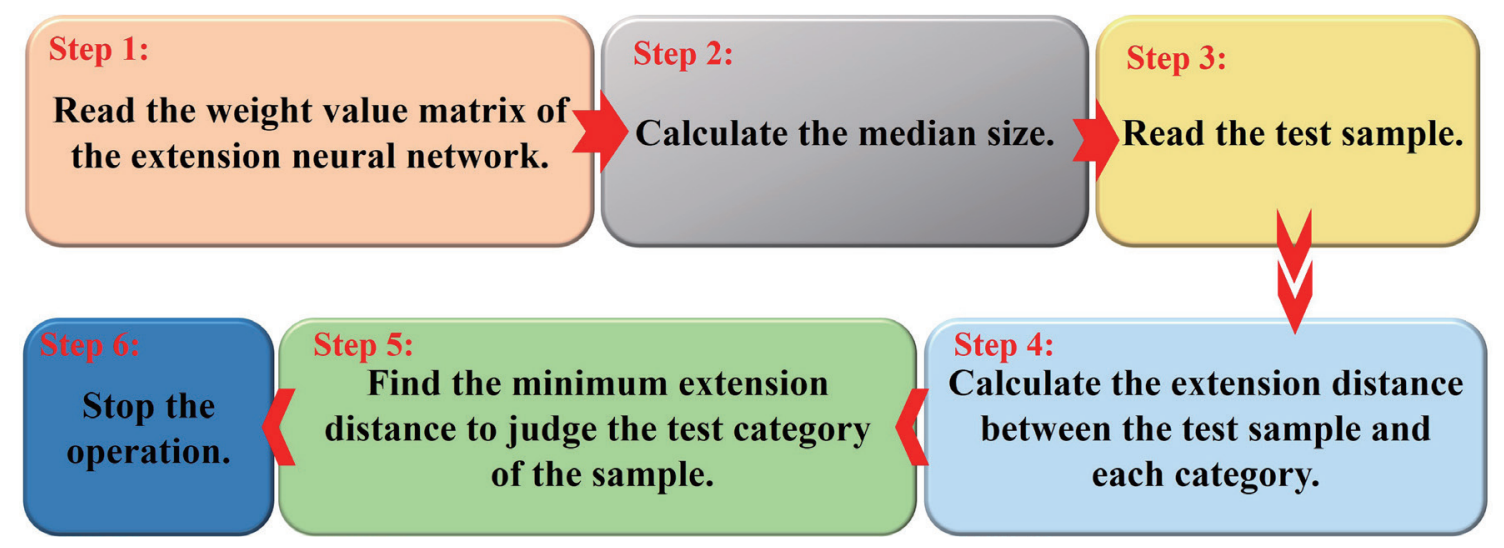

Fig. 14. (Color online) Identification calculus process of ENN. 


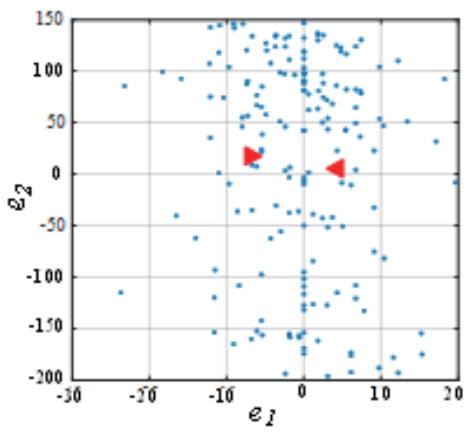

(a)

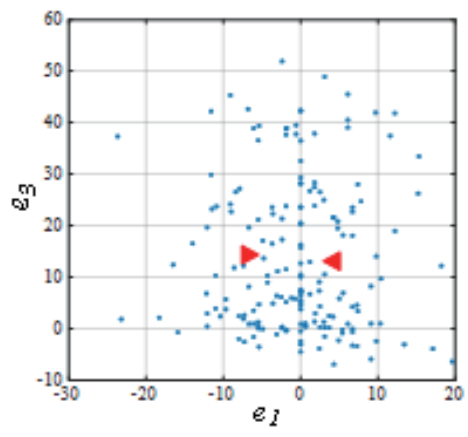

(b)

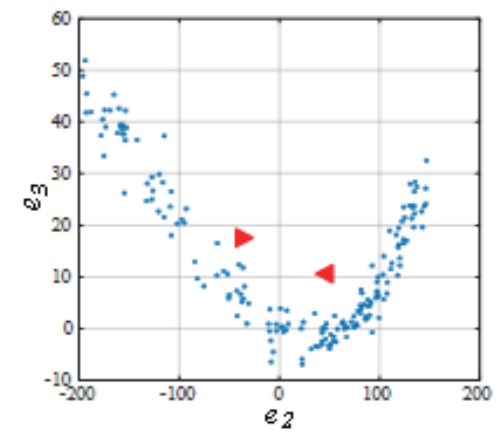

(c)

Fig. 15. (Color online) Lorenz chaotic dynamic error scatter diagrams. (a) $e_{1} e_{2}$, (b) $e_{1} e_{3}$, and (c) $e_{2} e_{3}$.

Table 2

Diagnosis results for different chaotic systems.

\begin{tabular}{lcc}
\hline Chaotic system & Recognition accuracy $(\%)$ & Sequence \\
\hline Lorenz $e_{2} e_{3}$ & 76.25 & 3 \\
Lorenz $e_{1} e_{3}$ & 82.5 & 2 \\
Lorenz $e_{1} e_{2}$ & 88.75 & 1 \\
\hline
\end{tabular}

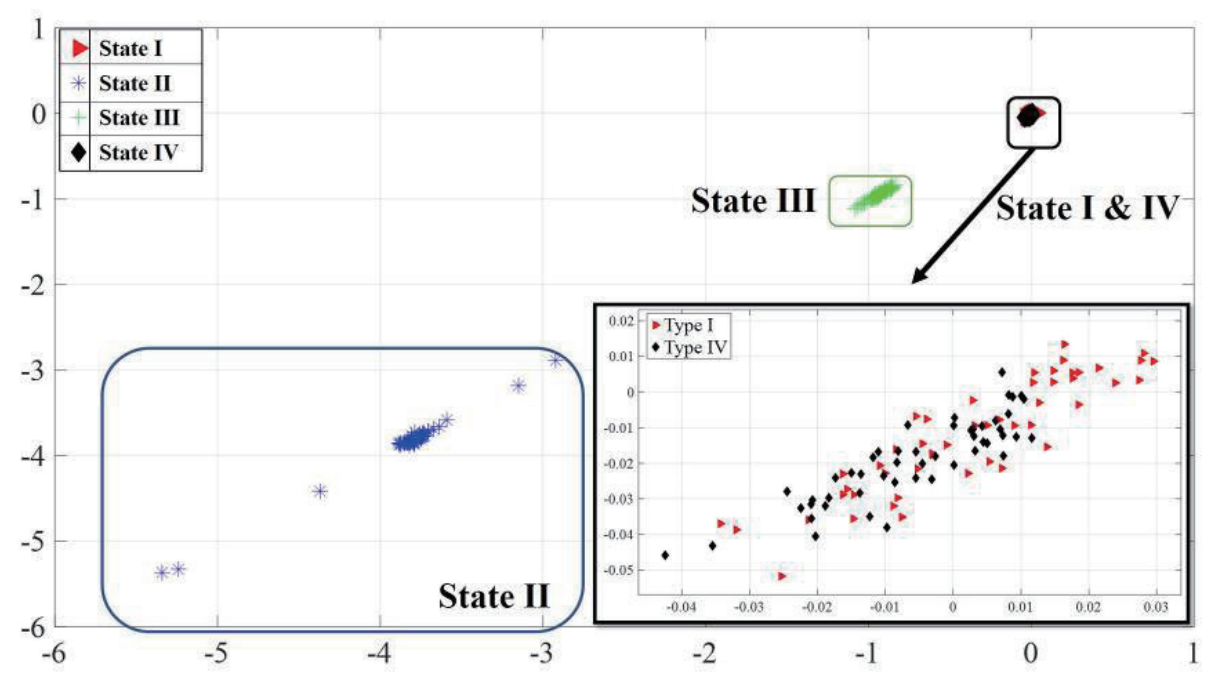

Fig. 16. (Color online) Left barycenter coordinate distribution graph of Lorenz chaotic system $e_{1} e_{2}$.

analysis. Figure 16 is the barycenter distribution diagram of Lorenz chaotic system $e_{1} e_{2}$. The results show that if $e_{1}$ and $e_{2}$ of the Lorenz chaotic system are selected as the eigenvalues of the chaotic barycenter, then the best clustering effect can be obtained in the coordinate distribution of the fault barycenter and the highest identification degree can be obtained in the ENN identification. 


\subsection{ENN fault identification}

The ENN model designed in this study uses the chaotic barycenter coordinate value of the chaotic dynamic error dispersion diagram of the blade vibration signal as identification data. There are 200 bits of measured vibration signal data and 50 bits of data for each state. The ENN model for identifying wind turbine blade states (i.e., the normal state and the three faults) is trained from 120 sets of chaotic barycenter coordinates, and each state has 40 sets of chaotic barycenter coordinates. To verify the effectiveness of the ENN model in identifying different states, 80 groups of chaotic barycenter coordinates are tested in this study, and each state has 20 groups of barycenter coordinates. Table 3 lists the results of ENN identification of the wind turbine blade states. The proposed method effectively identifies different wind turbine blade states with an identification accuracy of $88.75 \%$.

We compare the proposed method with three types of neural network commonly used in traditional fault identification: the back-propagation neural network (BPNN), learning vector quantization network (LVQN), and probabilistic neural network (PNN). The test environment is MATLAB 2020a with an Intel Core 17-9700 CPU @ 3.0 GHz processor, NVIDIA GeForce RTX 2080 SUPER display adapter, and Windows 10 operating system. Table 4 shows the experimental results. The PNN can complete the training of the classifier in the shortest time of $0.001 \mathrm{~s}$, followed by the proposed ENN (0.03 s), the BPNN (9.35 s), and the LVQN (19.79 s), which is the most time-consuming among the four detection methods. The ENN has $88.75 \%$ recognition accuracy, which is the highest among the four detection methods, followed by the PNN (78.75\%), LVQN (71.25\%), and BPNN (70\%). As a whole, although the PNN may complete the training faster, its identification accuracy is clearly less than that of the ENN. Although the proposed method is second only to the PNN in training time, its identification accuracy is $10 \%$ higher than that of the PNN.

Table 3

Results of identification of wind turbine blade states.

\begin{tabular}{lccc}
\hline State & $\begin{array}{c}\text { Number of test } \\
\text { patterns }\end{array}$ & $\begin{array}{c}\text { Number of accurate } \\
\text { patterns }\end{array}$ & $\begin{array}{c}\text { Recognition rate } \\
(\%)\end{array}$ \\
\cline { 1 - 3 } Normal & 20 & 17 & \multirow{2}{*}{88.75} \\
\cline { 1 - 3 } Blade rupture & 20 & 20 & \\
\cline { 1 - 3 } Blade screw fly-off & 20 & 14 & \\
\cline { 1 - 3 } Abnormal blade inclination angle & 20 & 14 & \\
\hline
\end{tabular}

Table 4

Comparison of identification performance between ENN and traditional neural monitoring methods.

\begin{tabular}{lccc}
\hline Detection method & $\begin{array}{c}\text { Accuracy of } \\
\text { training (\%) }\end{array}$ & $\begin{array}{c}\text { Training } \\
\text { time (s) }\end{array}$ & $\begin{array}{c}\text { Identification } \\
\text { accuracy (\%) }\end{array}$ \\
\hline Back-propagation neural network & 73.33 & 9.35 & 70.00 \\
\hline Learning vector quantization network & 85.83 & 19.79 & 71.25 \\
\hline Probabilistic neural network & 100 & 0.001 & 78.75 \\
\hline Extension neural network & 89.16 & 0.03 & 88.75 \\
\hline
\end{tabular}




\section{Conclusions}

This paper presented a fault identification method for wind turbine blades based on a chaotic system in which an ENN identifies four common states of wind turbine blades. It uses the Lorenz chaotic system to draw chaotic dynamic error scatter diagrams from the detection data as fault diagnosis samples, and employs the trained ENN weight model to identify the states. The performance of the ENN was compared with that of three different traditional neural networks: the BPNN, LVQN, and PNN, and the identification accuracy of the proposed method was found to be the highest at $88.75 \%$. We also constructed a remote human-machine interface to integrate the identification system with the monitoring system and develop a wind turbine fault diagnosis and monitoring system. The characteristics of the method proposed herein are as follows. (1) The combination of chaotic dynamic error scatter diagrams and the ENN is able to effectively identify the fault characteristics of blades. (2) The fault diagnosis human-machine interface developed by LabVIEW graphic control software also effectively identifies the states of wind turbine blades online, thus making the operation and maintenance of wind turbines more efficient.

\section{Acknowledgments}

The authors gratefully acknowledge the financial support of the Ministry of Science and Technology of Taiwan under contract numbers MOST 109-2221-E-167-009 and MOST 108-2221-E-167-018-MY2.

\section{References}

1 GWEC Global Wind Report: https://gwec.net/global-wind-report-2019 (accessed March 2020).

2 IEA World Energy Outlook 2020: https://www.iea.org/reports/world-energy-outlook-2020 (accessed October 2020).

3 RE100 International Initiative: https://www.there100.org/technical-guidance (accessed January 2021).

4 P. Zhang and D. Lu: Energies 12 (2019) 151. https://doi.org/10.3390/en12142801

5 K. Y. Oh, J. K. Lee, J. Y. Park, B. J. Kim, and J. S. Lee: IEEE Trans. Instrum. Meas. 64 (2015) 1726. https://doi. org/10.1109/TIM.2014.2381791

6 J. Yang, L. Zhao, Z. Lang, and Y. Zhang: 2018 10th Int. Conf. Modelling, Identification and Control (ICMIC) (2018) 1-6. https://doi.org/10.1109/ICMIC.2018.8529879

7 L. Wang and Z. Zhang: IEEE Trans. Ind. Electron. 64 (2017) 7293. https://doi.org/10.1109/TIE.2017.2682037

8 Y. Zhang, Y. Cui, Y. Xue, and Y. Liu: IEEE Access 8 (2020) 105094. https://doi.org/10.1109/ ACCESS.2020.2999783

9 H. Malik and S. Mishra: IET Renewable Power Gener. 11 (2017) 889. https://doi.org/10.1049/iet-rpg.2015.0382

10 S. D. Lu, M. H. Wang, and S. K. Chen: J. Low Freq. Noise Vibr. Act. Control 39 (2020) 665. https://doi. org $/ 10.1177 / 1461348419829668$

11 M. H. Wang, S. D. Lu, and M. J. Hsieh: Measurement 129 (2018) 227. https://doi.org/10.1016/j. measurement.2018.07.022.

12 E.N. Lorenz: J. Atmospheric Sci. 20 (1963) 130. https://doi.org/10.1175/1520-0469(1963)020<0130:DNF>2.0.CO;2

13 L. M. Pecora and T. L. Carroll: Phys. Rev. Lett. 64 (1990) 821. https://doi.org/10.1063/1.4917383

14 H. T. Yau and M. H. Wang: IET Renewable Power Gener. 9 (2015) 593. https://doi.org/10.1049/iet-rpg.2014.0269

15 B. Das: IEEE Trans. Power Delivery 21 (2006) 278. https://doi.org/10.1109/TPWRD.2005.852273

16 M. B. I. Reaz, F. Choong, M. S. Sulaiman, F. Mohd-Yasin, and M. Kamada: IEEE Trans. Power Delivery 22 (2007) 1979. https://doi.org/10.1109/TPWRD.2007.899774

17 J. C. Tovar: Proc. 2007 IEEE 22nd Int. Symp. Intelligent Control (IEEE, 2007) 24-29. https://doi.org/10.1109/ CCA.2007.4389200 
18 W. Cai: J. Sci. Exploration 3 (1983) 83.

19 M. H. Wang and C. Y. Ho: IEEE Trans. Power Delivery 20 (2005) 1939. https://doi.org/10.1109/ TPWRD.2005.848673

20 M. H. Wang and C. P. Hung: Proc. Int. Joint Conf. Neural Networks (2003) 399-403. https://doi.org/10.1109/ IJCNN.2003.1223379

21 M. H. Wang: IEE Proc. Generation, Transmission and Distribution (IEE, 2004) 503-508. https://doi. org/10.1049/ip-gtd:20040538

\section{About the Authors}

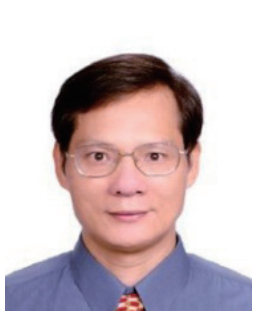

Meng-Hui Wang received his M.S. degree in electrical engineering in 1990 and his Ph.D. degree in the same field in 1994, both from National Taiwan University of Science and Technology. He came to National Chin-Yi University of Technology (NCUT) in August 1994 and is now affiliated with the Department of Electrical Engineering as a lifetime distinguished professor. His major areas of research include renewable energy systems, power systems, extension theory, and AI applications. He is a member of the Chinese Association of Artificial Intelligence (CAAI), the vice president of the Taiwan Education Society of Innovation \& Invention (TESII), and also the chairman of the 6th Intelligent Living Technology Association of Taiwan (ILTAT). He was the general chair of the 1st Intelligent Living Technology Conference (2006) and the honorary co-chair of the 2012 International Symposium on Computer, Consumer and Control (IS3C). (wangmh@ncut.edu.tw)

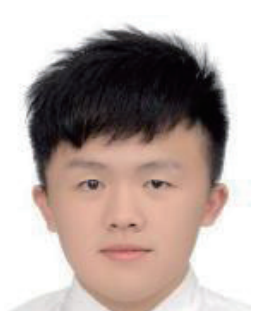

Cheng-Che Hsieh received his M.S degree from the Department of Electrical Engineering, National Chin-Yi University of Technology, Taichung City, Taiwan, in 2021. His main research includes artificial engineering and fault diagnosis. (a0955381508@gmail.com)

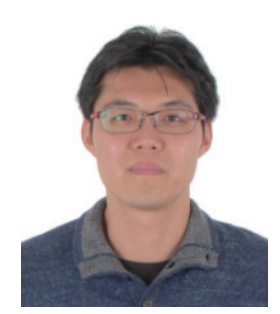

Shiue-Der Lu received his M.S. degree from Chung Yuan Christian University, Taoyuan City, Taiwan, in 2006 and his Ph.D. degree from the National Taiwan University of Science and Technology, Taipei City, Taiwan, in 2013, both in electrical engineering. From 2012 to 2016, he was a researcher at the Industrial Technology Research Institute. From 2016 to 2018, he was an assistant researcher at the Department of Electrical Engineering, Chung Yuan Christian University. Since 2018, he has been with the Department of Electrical Engineering, National Chin-Yi University of Technology, Taichung City, Taiwan, where he is currently an associate professor. He is also the secretary-general of the 6th Intelligent Living Technology Association of Taiwan (ILTAT). His research interests include renewable energy, power quality, damage diagnosis of wind power generators, and artificial intelligence applications in power systems. (sdl@ncut.edu.tw) 